\title{
Editorial Special Issue: PHM for Railway Systems and Mass Transportation
}

\author{
John Andrews, Thomas Böhm, Pierre Dersin, Jörn C. Groos, and René Schenkendorf
}

The railway and mass transportation system is composed of industrial goods with substantial capital investments and long life cycles. This applies to rolling stock like trains, locomotives, wagons, and even more to the infrastructure like signaling, catenary, tracks, bridges, and tunnels. The lifespan of rolling stock is 30 to 40 years while the infrastructure is used 30 to 60 years even more than 100 years in case of tunnels and bridges. As in other industrial goods, the cost drivers are determined in the early design phases but realized mainly during a long time of operation. Maintenance is one of the main cost drivers but essential to a reliable, capable, and above all - safe operation.

Nowadays, depending on the country about 30 to 60 percent of the life cycle costs of railway rolling stock and infrastructures are made up by maintenance costs. In addition, rising demands on railroad infrastructure operators regarding profitability and punctuality call for advanced concepts of Prognostics and Health Management (PHM).

Condition-based preventive maintenance aims at strengthening the rail mode of transport through an optimized scheduling of maintenance actions based on the actual and prognosticated condition. Prerequisite, therefore, is the almost continuous condition monitoring for thousands of kilometers of railway tracks as well as ten thousands of technical systems and sub-systems. The rapidly expanding possibilities for embedded sensors in all types of technical components as well as in-line railway vehicles are the key enabler for conditionbased preventive maintenance in large and distributed railway networks. This Special Issue of IJPHM solicits papers that discuss the development of advanced sensor-based condition monitoring, smart data management, intelligent diagnostic data analysis, degradation models, condition prognosis and maintenance scheduling for railway systems. All systems considered benefit from PHM as summarized below.

Given the complex nature of PHM, it is good to start with an overview. The paper by Atamuradov, Medjaher, Dersin, Lamoureux \& Zerhouni gives a broad overview of PHM using examples not only from railway but also from other industries. They wrap up the different challenges scientists and practitioners may face when working on the topic. Their description of the essential tasks to implement PHM and the introduction of the most important norms for each task guide through the complex matter. Further, the authors explain and discuss the advantages and disadvantages of model-based, datadriven and hybrid approaches in PHM for various applications and methods, e.g., Particle Filter in crack propagation, or Hidden Markov Models in hydraulic pumps. Their review goes into more detail with a case study on bogie diagnostics and prognostics.

Condition-based maintenance (CBM) of rotating machinery is applied in many industries; mass transportation is no exception. The paper by Ashasi-Sorkhabi, Fong, Prakash, and Narasimhan presents an outline of the CBM program and a field pilot study on the gearbox in an automated cable-driven people mover (APM) system at Torontos Pearson airport. Fault detection is performed in real-time using vibration data from the APM gearbox with the baseline condition established by time-domain condition indicators. For prognostics, these indicators are utilized for degradation modeling and subsequent Remaining Useful Life (RUL) estimation using random coefficient and stochastic models. Parameter estimation is undertaken using a hierarchical Bayesian approach. While their case study primarily focuses on a cable-driven APM gearbox, the underlying theory and the tools developed to undertake diagnostics and prognostics tasks are broadly applicable.

For high-speed tilting trains, the correct functioning of the tilt angle measurements is crucial to ensure safe operation. For this reason, two sensors mounted at the front and the rear of each vehicle are part of the train control system. The detection of a discrepancy between the two sensors of a vehicle immediately disables the tilting function of the entire train to avoid critical safety situations. An integrated health monitoring framework to identify faulty sensors is presented in the paper by De Martin, Dellacasa, Jacazio, and Sorli. This framework allows reducing the negative impacts on train operation due to speed reductions caused by the failure of a single sensor. The framework is based on a sophisticated physical model of the control system and validated with experimental mission profiles on the Lichtenfels - Saafeld and Battipaglia - Reggio Calabria routes.

Air leakage in braking pipes on trains lead to braking issues train delays, respectively. The author Wee presents a data- 
processing framework for air leakage detection which predicts the severity of air leakages simultaneously. Thus, based on the severity level proper action plans can be implemented. By evaluating operating data of Dutch Railways the estimation of the remaining useful life of the air braking pipe for given severity levels is shown. The results have demonstrated that most air leakages can be detected one to four weeks before the braking failure allowing targeted intervention strategies.

Controlling the right friction levels between the wheel and rail in railway systems is critical to ensure a reliable traction. At low friction levels, trains lose traction and cause track damages. At high friction levels, excessive wear of the rail-surface can be expected. To balance the friction level, so-called friction modifiers (carrier with particles) are used. In their work, the authors Oomen, Bosman, and Lugt analyze experimentally three commercial friction modifiers regarding their friction characteristic and wear behavior. The results reveal that the level of friction is a function of total rolling distance, effective sliding length, and sum velocity. The most dominant factor depends on the friction modifier and the working mechanism for friction stabilization. Moreover, wear rates are dependent on the type of friction modifier used.

Fiber Optic Sensing (FOS) has become a popular technique to monitor large distributed structures. The authors Arakaki, Raghavan, and Schuh push the boundaries for the application of FOS in railways. Their high resolution, low-cost optical readout could obtain a sensitivity of less than $90 \mathrm{fm}$ at frequencies up to $80 \mathrm{kHz}$ (claimed $>10 \mathrm{x}$ the state-of-the-art), as well as be scaled up to monitor many multiplexed sensors. This enabled them to overcome issues of past work. In particular, they correctly distinguished a broken rail from insulation joints and detected rail surface abrasion in their laboratory setting.

Switches (or turnouts) are essential for a flexible operation but often responsible for high maintenance cost and delays. The manuscript by Böhm introduces an RUL prediction for switches based on several data sources, e.g., in service continuous condition monitoring, weather, failure documentation. The RUL prediction is transformed into a classification problem of multiple classes with the effect of reducing uncertainty. The Artificial Neural Networks (aNN) and Support Vector Machines (SVM) are used to predict the RUL in the form of classes. Showing that the performance of aNN and SVM heavily depends on their parametrization, the author derives those parameters maximising the prediction results. This enables one of the two classification techniques to precisely reveal future failures of the switch engine early enough to prevent them.

The manuscript by Brahimi, Medjaher, Leouatni, and Zerhouni provides a comprehensive review of the current state of PHM for railway overhead contact lines. This includes the key sensors and monitoring approaches as well as the algorithms for detection, diagnosis, and prognosis. The authors present the design context of a PHM solution for overhead contact lines and discuss the critical system components as well as failure modes. Based on state of the art principles and the technical needs for predictive maintenance the recent research challenges are outlined to improve PHM for overhead contact lines further.

In their work, the authors Palmqvist, Olsson, and Hiselius study and quantify how weather, timetable, operational and infrastructure related variables influence the punctuality of the railway system. The study is based on a dataset containing detailed timetables and records of all 32.4 million train movements for all trains in Sweden during the year of 2015, over 1.1 million departures, a comprehensive register of over 80000 infrastructure elements, and almost 87 million weather observations. After identifying the most influencing factors on the punctuality intervention strategies can be derived regarding timetables, change of operational parameters and infrastructure design. This paper presents solutions to improve the railway system that go beyond diagnostics and prognostics to round up the possibilities of PHM.

Besides the technical aspects, the paper by Singh, Majumdar, and Kyriakidis analysis human errors occurring during railway maintenance activities. In detail, the human error assessment and reduction technique (HEART) is employed to determine the probability of human errors for typical maintenance tasks, while fault tree analysis is used to define potential mistakes throughout the maintenance process. Their findings might be a valuable source to revise policies and guidelines in terms of improved and secure railway maintenance tasks.

We, the Guest Editors, are confident that this Special Issue on PHM for Railway Systems and Mass Transportation provides exciting insights into PHM solutions. The dissemination of the presented approaches and results will advance the state of research and development in this field. The proposed concepts hopefully lead to a safer, more reliable and cost-efficient railway transportation. We would like to express our sincere appreciation to the authors for their efforts in preparing and submitting these outstanding manuscripts. Likewise, we would like to thank the reviewers for their time and thoughtful feedback on the submitted papers.

JOHN ANDREWS Guest Editor

Royal Academy of Engineering and Network Rail Chair of Infrastructure Asset Management

Director of the Lloyds Register Foundation Resilience Engineering Research Group University of Nottingham, United Kindom 
ThOMAS BöHM Guest Editor

Chief Data Scientist

Konux

Munich, Germany

PIERE DERsin Guest Editor

PHM Director

ALSTOM Digital Mobility

St-Ouen, France

JÖRn C. GROOS Guest Editor

Team Leader

Institute of Transportation Systems

German Aerospace Center (DLR)

Braunschweig, Germany

RENÉ SCHENKENDORF Guest Editor

Postdoctoral Researcher / Team Leader

Institute of Energy and Process Systems Engineering

TU Braunschweig, Germany

\section{BIOGRAPHIES}

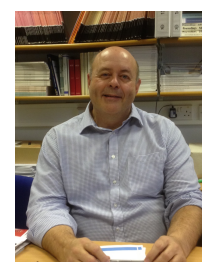

John Andrews has a degree in Mathematics from the University of Birmingham and a $\mathrm{PhD}$ in Mechanical Engineering. Prior to his appointment at the University of Nottingham he worked for 20 years at Loughborough University where his final post was Professor of Systems Risk and Reliability The prime focus of his research has been on methods for evaluating the system resilience, unavailability, unreliability and risk. Recent work has concentrated on degradation modelling and the effects of maintenance, inspection and renewal on railway asset performance. In 2005, he founded the Proceedings of the Institution of Mechanical Engineers, Part O: Journal of Risk and Reliability of which was the Editor-in-chief for 10 years. He is also a member of the Editorial Boards for 6 other international journals in this field including: Reliability Engineering and System Safety and Quality and Reliability Engineering International. In 2011 he co-edited, along with Christophe Berenguer and Lisa Jackson, Maintenance Modelling and Applications, published by ESReDA (European Safety Reliability and Data Association). John has over 300 publications in the field of Risk, Reliability and Maintainability Engineering. In recent years John has been awarded the Moss Prize, the Ludwig Mond Prize, the Donald Julius Groen Prize and the Charles Sharpe Beecher Prize from the IMechE.

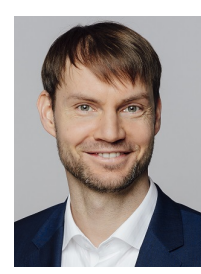

Thomas Böhm (Boehm) studied at University of Magdeburg in Germany and the EPFL Lausanne in Switzerland. He holds a Master in Computer Science in Engineering and has joined the DLR (German Aerospace Center) Institute of Transportations Systems in 2007. Since then, his main field of research is condition based maintenance and health management using statistics and data mining. In 2010, Thomas became leader of the Life Cycle Management team with whom he further advanced the topic of efficient, predictive maintenance of railway infrastructure. He has several publications in this field and is session chairman of the International Congress of Condition Monitoring and Diagnostic Engineering Management. Thomas worked in and led several international and national projects as well as development projects for diagnostic and prognostic algorithms for Deutsche Bahn and its asset monitoring platform DIANA. In 2015, he became Chief Officer and Head of the Department Data Management and Knowledge Discovery. In this role, Thomas was responsible for sensor system development, data management, monitoring, and situation interpretation for road traffic and autonomous driving as well as railway system. Since 2017 , he is the Chief Data Scientist at KONUX, a company focusing on analytics and predictive maintenance.

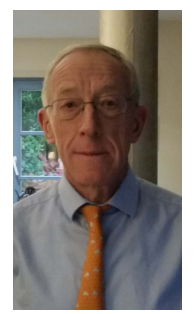

Pierre Dersin was born and raised in Belgium and graduated from the Massachusetts Institute of Technology (MIT) with a Ph.D. in Electrical Engineering after receiving a Masters degree in Operations Research also from MIT. He worked on reliability of large electric power networks, as part of the Large Scale System Effectiveness Analysis Program sponsored by the US Department of Energy, from MIT and Systems Control, Inc. After some time with FABRICOM (Belgium and U.S), involved with fault diagnostic systems for factory automation, he joined, in 1990, ALSTOM Transport in Paris, where he has occupied several positions, mainly involved with RAMS and Maintenance, and he founded the RAM Center of Excellence . He is now RAM (ReliabilityAvailability-Maintainability) Director and PHM (Prognostics \& Health Management) Director of ALSTOM Digital Mobility. He has contributed a number of communications and publications in scientific conferences and journals in the fields of RAMS, PHM, automatic control and electric power systems (including IEEE Transactions on Automatic Control, IEEE Transactions on Power Apparatus \& Systems, ESREL, almost every RAMS Symposium since 2008, several of the French Lambda-Mu conferences, the 2012 IEEE-PHM Conference and WSC 2013). He was a keynote speaker at the European PHM Conference (Nantes) in 2014 and at the Prognostics and system Health Management conference in Harbin (China) in 2017. He served on the IEEE Reliability Society AdCom from 2012 to 2017 and as Vice-President, Technical Activities, in 2017. He chairs the IEEE Reliability Society Technical Committee on Systems of Systems, and is also a member of the IEEE Future Directions Committee. He is the lead author of 4 chapters, including one on PHM, in the forth- 
coming Handbook of RAMS in Railways: Theory \& Practice (Taylor \& Francis).

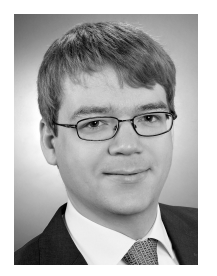

Jörn C. Groos received his diploma in geophysics from the University of Karlsruhe, Germany, in 2007 and his Dr. rer. nat. from the faculty of physics of the Karlsruhe Institute of Technology in 2010. Since 2014 he is with the German Aerospace Center DLR at the Institute of Transportation Systems in Braunschweig, Germany. Jörn is leading the Asset and System Monitoring group within the Department Data Management and Knowledge Discovery. The German Aerospace Center is Associate Member of the European Joint Undertaking Shift2Rail and Jörn is responsible for the DLR contributions to the Innovation Programme 3 Cost-Efficient and Reliable High-Capacity Infrastructure. His current research interests include data acquisition, data analysis, and machine learning for condition monitoring of railway assets.

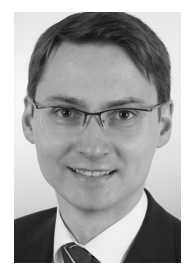

René Schenkendorf received his Dipl.-Ing. and Dr.-Ing. in Engineering Cybernetics from the Otto-von-Guericke-University Magdeburg, Germany in 2007 and 2014, respectively. From 2007 until 2012 he had been a Ph.D. student at the Max Planck Institute for Dynamics of Complex Technical Systems in Magdeburg, Germany. From

2013 until 2016 he was with the German Aerospace Center at the Institute of Transportation Systems in Braunschweig, Germany. Since 2016 René is the head of the Process Systems Engineering group at the Institute of Energy and Process Systems Engineering at the Technische Universität Braunschweig, Germany. His current research interests include data analysis, statistical process control, uncertainty analysis, and modeling in PHM. 